\title{
A New Enhanced Variation of TF-IDF Seheme for Arabic Text Classification
}

\author{
Fawaz S. Al-Anzi ${ }^{1}$, and Dia AbuZeina ${ }^{2}$
}

\begin{abstract}
Text Classification (TC) is a popular information retrieval (IR) technique that mainly employs features selection, features reduction, and features weighting techniques. The most common technique for weighting textual features is the Term Frequency-Inverse Document Frequency (TF-IDF) scheme. However, the standard TF-IDF can be enhanced as presented in the literature. In this paper, we propose a new variant of the TF-IDF by considering the small words (such as two, three, and four characters). The new method proposes modifying the weight of each word based on its length. To investigate the performance, a corpus contains 4,000 documents of ten topics (400 document for each topic) was used. The corpus contains more than 2 million words with about 140,000 unique words. The Testing set contains 400 documents, 40 documents for each topic. The latent semantic indexing (LSI) and the cosine similarity measure were used for features reduction and text classification, respectively. The experimental results show that modifying the weights according to the word's length significantly enhance the accuracy by $3.75 \%$.
\end{abstract}

Keywords-Arabic Text; Classification; Features Reduction; Weighting; Latent Semantic Indexing

\section{INTRODUCTION}

$\mathrm{T}$ ERM Frequency-Inverse Document Frequency (TF-IDF) is a popular weighting scheme that is used in information retrieval (IR) and the related text classification (TC) domain. Traditionally, TC problems use vector space model (VSM) as a method to represent documents (or texts) as vectors, [1]. The documents vectors are then weighted to indicate the important of each term in the documents vectors. The TF-IDF method uses two metric to find the weigh; TF that indicates the frequency that a term appears in the document, while IDF represents the inverse of the tem occurrences in entire training documents. TF-IDF is the most common representation term weighting technique that is widely used to determine the significant words (features) in the text, [2]. The TF-IDF method has been widely investigated to measure its performance. For, example, Reference [3] indicated that TFIDF method seems very appropriate for IR; however, it is not clear if it is the best choice for TC problems.

In this paper, we propose a new variant of TF-IDF that consider reweighting small words that include two or three

Fawaz S. Al-Anzi ${ }^{1}$ is with Department of Computer Engineering, Kuwait University, Kuwait.

Dia AbuZeina ${ }^{2}$ is with Research Sector, Kuwait University, Kuwait. characters. For example, the Arabic language has a set of prepositions that have relatively low discriminative power as they spread out all over the documents. Even TF-IDF already reduces the weights of such common small words, this study propose to farther reducing the weights according to the word's length. One technique to handle common words in TC problem is to group them in a set called Stop words or Stop list to have them excluded when preparing the corpus dictionary. Another technique is to use the TF-IDF method to reduce the effect of such common words during the classification. Even though that these technique have well success in IR, however, researching continues to handle the common words problem as we demonstrate in this work. The latent semantic indexing (LSI) and the cosine similarity measure were used for features reduction and text classification, respectively.

In the next section, we present the motivation of this work, section III have the literature review, followed by the experiments setup in section IV. We present the results in section $\mathrm{V}$ and the conclusion in section VI.

\section{THE MOTIVATION}

Pattern recognition performance is primarily depends on the real differences (dissimilarities) among the used patterns. That is, if most patterns have a high percentage of common features how the classification methods can differentiate between the pattern classes or the text topic as in our case. Accordingly, the commons words in text classification problems produces suboptimal performance. Fig. 1 shows that the Training set used in this work has $12 \%$ of 2 characters words, and $15 \%$ of 3 characters words.

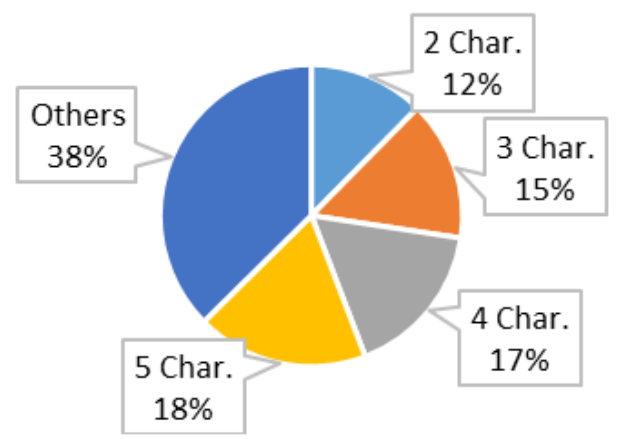

Fig. 1 Words Distribution Statistics in the Trining Set

These small words proportions contain common words that 
can be discarded to enhance the performance as well as to reduce the ambiguity and computational complexity. The work in this paper is not exclusive for the Arabic language; English has also small words (e.g. the, or, in, etc.) that probably have the same characteristics as Arabic, of course, form classification point of view. Hence, this work is based on discarding or considering such small words in the weighting process to reduce its effect in the classification process. In this work and for a comparison purpose, the performance was measured on a series of words lengths that have more than three characters such as four, five, and six characters.

\section{LITERATURE REVIEW}

The literature review shows that TF-IDF has been widely used in TC. In this literature, we focus on the usage of this weighting method for the Arabic language. In the literature, it has been noticed that some researchers mixed this weighing scheme with the features selection and features reduction methods. Standard TF-IDF was used in the following Arabic text related systems (i.e. classification, summarization, clustering, etc.): [4] - [36].

Away from the Arabic language TC research, some studies proposed modification of TF-IDF such as what introduced in [3] to use statistical confidence intervals to estimate the importance of a word for a specific categorization problem. Reference [37] proposed a weighting approach: TF-IDF-Ci where $\mathrm{Ci}$ is added to express the differences between classes on the base of original TF-IDF. Reference [38] proposed three weighting schemes that exploit the class space density based on the class distribution in the whole document set as well as the class documents set. Reference [39] summarized some of the term weighting formulas. However, with the noticeable success of TF-IDF, Reference [40] presents two criticisms of TF-IDF. The first one is that TF-IDF is too 'ad hoc', and the second one is the dimensionality that leads to huge computation.

The literature review shows that small words has not been considered in the TC problems. Hence, improving the TF-IDF weighting scheme using the small words is the main essence of our proposed work.

\section{EXPERIMENT SETUP}

\section{A.Data Set}

To investigate the proposed method, a Training Set of 4,000 documents was created. The documents belong to 10 different categories. The Testing set contains 400 documents. The corpus contains more than 2 million words. Table I shows the corpus information.
TABLE I

THE CORPUS INFORMATION

\begin{tabular}{|l|l|l|l|}
\hline$\#$ & \multicolumn{1}{|c|}{ Category } & \multicolumn{1}{c|}{ \# Documents } & \multicolumn{1}{c|}{ \# Words } \\
\hline 1 & Health & 400 & 218,214 \\
\hline 2 & Economy & 400 & 181,366 \\
\hline 3 & Crimes and courts & 400 & 172,145 \\
\hline 4 & Education & 400 & 259,127 \\
\hline 5 & Technology & 400 & 209,319 \\
\hline 6 & Sports & 400 & 168,934 \\
\hline 7 & Tourism & 400 & 270,142 \\
\hline 8 & Islam and Sharia & 400 & 242,943 \\
\hline 9 & Parliament & 400 & 182,503 \\
\hline 10 & Political Affairs & 400 & 222,504 \\
\hline & Total & 4,000 & $2,127,197$ \\
\hline
\end{tabular}

\section{B.Preprocessing}

For the entire corpus (4,000 documents for training and 400 document for testing), a preprocessing step is performed to prepare the text for the classification process. Therefore, cleaning the text has the following four steps: The Stop list is declared to remove the common words such as the newspaper name. The ignore characters are declared. It includes $\{\sim, `$, , @ , \#, $£, €, \$, \%,{ }^{\circ}, \wedge, \&, *,(),,-,,_{-},+,=, », \ll,\{$, $,[],, \mid, \backslash, /,:, ;, 0,1,2,3,4,5,6,7,8,9\}$. The character "I)" is replaced by "l", and "!" by "". Finally, a single character is deleted wherever appears in the corpus.

\section{Proposed TF-IDF variation}

The proposed method includes two parts; the first part is to discard the words that are less than certain threshold, and the second part is to consider the small words in the traditional weighting scheme TF-IDF to produce a new variant of the classical TF-IDF formula:

$$
w_{i, j}=t f_{i, j} \times \ln \left(\frac{N}{d f_{i}}\right)
$$

Where wi, $\mathrm{j}$ is the weight of word $\mathrm{i}$ in document $\mathrm{j}, \mathrm{N}$ is the total number of documents in the corpus (i.e., The Training Set), tfi,j is the word frequency of word $i$ in document $j$ and dfi is the document frequency of word $\mathrm{i}$ in the corpus. The proposed method suggests modifying the classical formula as the following format:

$$
w_{i, j}=t f_{i, j} \times \ln \left(\frac{N}{d f_{i}}\right) \times \ln (\text { length }(\text { word }))^{3}
$$

The reason of choosing ln function is the characteristic of this function that penalizes the small words more than the long words. Hence, the change of the words weights will be gradually decreases as we move from small to the long words as shown in Fig. 2. The Figure shows that while small words noticeably affected by the change, however, the long one are slightly affected. For example, If the word length is two, then the standard TF-IDF will be reduced by multiplying the word's weight by this value $(\ln 2 * \ln 2 * \ln 2)=0.333$. 


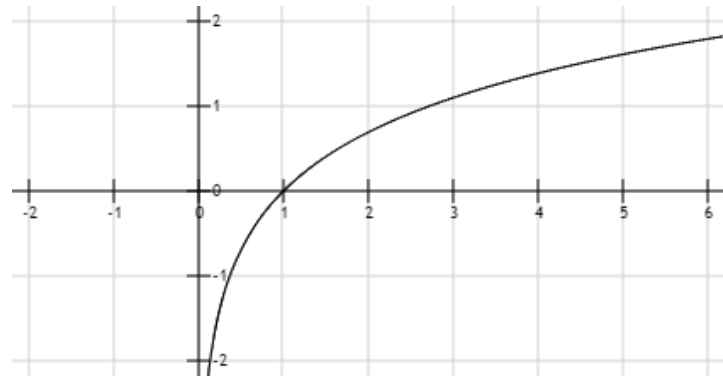

Fig. 2 Ln Function Graph

We emphasize that the proposed method does not reduce the weights for all lengths, but it also increases the weight for some words such the word of length 4 as the weight will be increased when multiplying it by the value $2.664(\ln 4 * \ln 4 *$ $\ln 4$ ). The power 3 in the proposed formula is selected based on the experimental investigation.

The implementation of the proposed method include the following steps:

Step 1: Using python, the LSI technique is used to generate the term-by-document matrix according to the VSM model.

Step 2: The term-by-document matrix is weighted using the standard TF-IDF method.

Step 3: The Singular Value Decomposition (SVD) method is used to truncate the term-by-document matrix to produce the documents features vectors. Matlab was used for this step.

Step 4: The cosine similarity measure is used to classify the documents vectors of the Testing set. Matlab was used.

Step 5: The performance is measured.

Step 6: The steps from 1 to 5 are repeated using the proposed weighting scheme.

\section{RESULTS}

The experiments were conducted for two cases, small words discarding and small words reweighting. In all experiments, the document frequency (DF) features selection method was set at 25 . That is, a word will be included in the dictionary (i.e. classification process) if it appears in 25 or more different documents.

\section{A. Discarding Small Words}

The performance was measured for discarding the word of less than or equal these thresholds: $2,3,4,5$, and 6 . For each experiments, the investigation include the rank $\mathrm{k}$ value form 10 up 100 and report the highest accuracy as shown in Table II. In the table, the first highlighted row shows the baseline performance that has not discarding any small words.

TABLE II

THE ACCURACIES OF DISCARDING SMALL WORDS

\begin{tabular}{|c|c|c|c|}
\hline$\#$ & Threshold & Highest at k equal & Accuracy (\%) \\
\hline & baseline & 40 & 83.50 \\
\hline 1 & $<=2$ & 38 & 84.75 \\
\hline 2 & $<=3$ & 36 & 83.50 \\
\hline $\mathbf{3}$ & $<=\mathbf{4}$ & $\mathbf{3 1}$ & $\mathbf{8 5 . 7 5}$ \\
\hline 4 & $<=5$ & 47 & 85.00 \\
\hline 5 & $<=6$ & 46 & 82.25 \\
\hline
\end{tabular}

Table II shows that discarding small words enhances the performance by $(85.75-83.50) 2.25 \%$ at $k=31$. This means that a better accuracy is achieved with less dimensions of the features vectors (31 instead of 40) and therefore less computational time. It also shows that discarding the words less than or equal 6 will reduce the performance as the dictionary start losing significant words.

\section{B. Keeping Small Words with Reweighting}

The proposed weighing formula was implemented to find the effect of penalizing small words of overall performance. This option might be better (practically found better) than discarding small words since many keys phrases for some category are small words such as: $\{$ فن , كرة, دم, بنك , نفط with the meaning: $\{$ art, soccer, blood, bank, oil $\}$. Hence, the experiments in this section do not discard the small words. Fig. 3 shows the accuracies for $\mathrm{k}=10$ to 100 . The bold line indicates the accuracy of the proposed method. The maximum accuracy was $86 \%$ and it was achieved at $\mathrm{k}=53$. This means that reweighing small words is better than discarding them since the maximum accuracy $(86.00 \%)$ was better than all cases when discarding small words as shown in Table II.

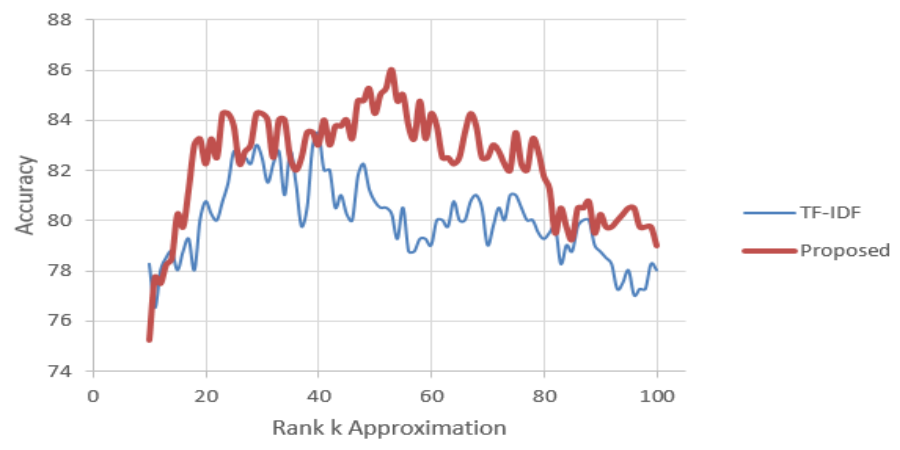

Fig. 3 The Proposed Method Accuracy

To find the best spot of accuracy, we repeat the experiments conducted in the previous section but with the proposed weighting method. Therefore, we consider discarding and reweighting during all experiments. The results reported in Table III.

TABLE III

THE ACCURACIES OF DISCARDING AND WEIGHTING

\begin{tabular}{|c|c|c|c|}
\hline$\#$ & Threshold & Highest at k equal & Accuracy $(\%)$ \\
\hline 1 & $<=2$ & 38 & 85.25 \\
\hline $\mathbf{2}$ & $<=\mathbf{3}$ & $\mathbf{4 2}$ & $\mathbf{8 7 . 2 5}$ \\
\hline 3 & $<=4$ & 41 & 86.25 \\
\hline 4 & $<=5$ & 27 & 86.25 \\
\hline 5 & $<=6$ & 23 & 82.25 \\
\hline
\end{tabular}

Hence, the experiments shows that the best performance was achieved when discarding the words that are less than or equal 3 , and on the same time implementing the proposed weighting scheme. The highest accuracy $(87.25 \%)$ was achieved at $\mathrm{k}$ $=42$. Therefore, this study indicates that handling small words could improve the performance by (87.25-83.5) $3.75 \%$.

To investigate whether the proposed method significantly enhances the performance, the performance detection method proposed by [41] was used. The confidence interval $[\varepsilon l, \varepsilon u]$ 
has to be compute at the first place. Fig. 4 shows how to find the confidence interval. $\mathrm{N}$ is set to the value 400 , the number of documents in the Testing set. If the changed classification error rate is outside the confidence interval, these changes can be interpreted as statistically significant. Otherwise, they were most likely caused by chance. We used $90 \%$ as a level of confidence. We also used the error probabilities $(\hat{\mathcal{E}})$ of the TF.IDF method (baseline), as (100-83.50) 16.50\% as reported in Table II. Since we used $90 \%$ as a level of confidence, $\mathrm{z}$ is equal 1.645 from the standard normal distribution. It might be interpreted as a $90 \%$ probability that a standard normal variable, $\mathrm{z}$, will fall between -1.645 and 1.645 .

$$
\begin{aligned}
& \mathcal{E}_{l}=\frac{N}{N+z^{2}}\left(\hat{\mathcal{E}}+\frac{z^{2}}{2 N}-z \sqrt{\frac{\hat{\mathcal{E}}(1-\hat{\mathcal{E}})}{N}+\frac{z^{2}}{4 N^{2}}}\right) \\
& \mathcal{E}_{u}=\frac{N}{N+z^{2}}\left(\hat{\mathcal{E}}+\frac{z^{2}}{2 N}+z \sqrt{\frac{\hat{\mathcal{E}}(1-\hat{\mathcal{E}})}{N}+\frac{z^{2}}{4 N^{2}}}\right)
\end{aligned}
$$

Fig. 4 Confidence Interval Calculation Formula

The confidence interval is found to be [16.50\%-2.83, $16.50 \%+3.28] \rightarrow[13.67 \%, 19.78 \%]$. Since the error probabilities using the proposed method is $12.75 \%$ (100$87.25 \%$ ), we consider that using small words significantly outperform the standard TF.IDF features as $12.75 \%$ is outside the confidence interval.

\section{CONCLUSION}

This study explores the effect of discarding and reweighting small words on the Arabic language text classification. The study reveals that the performance can be enhanced using both cases; discarding and reweighting the small words. The study also shows that the combination of these two cases has the highest performance. The study also exposes that the traditional TF-IDF is not the optimal choice as it can be enhanced when considering the small words as reported in this research.

\section{ACKNOWLEDGMENT}

This work is supported by Kuwait Foundation of Advancement of Science (KFAS), Research Grant Number P11418EO01 and Kuwait University Research Administration Research Project Number EO06/12.

\section{REFERENCES}

[1] Salton, G., Wong, A., \& Yang, C. S. 1975. A vector space model for automatic indexing. Communications of the ACM, 18(11), 613-620. http://dx.doi.org/10.1145/361219.361220

[2] Choi, Dongjin, et al. "Text analysis for detecting terrorism-related articles on the web." Journal of Network and Computer Applications 38 (2014): 16-21.

http://dx.doi.org/10.1016/j.jnca.2013.05.007

[3] Soucy, Pascal, and Guy W. Mineau. "Beyond TFIDF weighting for text categorization in the vector space model." IJCAI. Vol. 5. 2005.

[4] Ghwanmeh, Sameh H. "Applying Clustering of hierarchical K-meanslike Algorithm on Arabic Language." International Journal of Information Technology3.3 (2005).
[5] Syiam, Mostafa M., Zaki T. Fayed, and Mena B. Habib. "An intelligent system for Arabic text categorization." International Journal of Intelligent Computing and Information Sciences 6.1 (2006): 1-19.

[6] Al-Shalabi, Riyad, Ghassan Kanaan, and M. Gharaibeh. "Arabic text categorization using kNN algorithm." Proceedings of The 4th International Multiconference on Computer Science and Information Technology. Vol. 4. 2006.

[7] Al-Kabi, M., and S. Al-Sinjilawi. "A comparative study of the efficiency of different measures to classify Arabic text." University of Sharjah Journal of Pure and Applied Sciences 4.2 (2007): 13-26.

[8] Hmeidi, Ismail, Bilal Hawashin, and Eyas El-Qawasmeh. "Performance of KNN and SVM classifiers on full word Arabic articles." Advanced Engineering Informatics 22.1 (2008): 106-111.

http://dx.doi.org/10.1016/j.aei.2007.12.001

[9] Sankarapani, M. K., Basnet, R. B., Mukkamala, S., Sung, A. H., Ribeiro, B., \& Coimbra, C. (2008, March). Translation Based Arabic Text Categorization. InProceedings of Second International Conference on Information Systems Technology and Management, Dubai.

[10] Althubaity, A., Almuhareb, A., Alharbi, S., Al-Rajeh, A., \& Khorsheed, M. (2008). KACST Arabic text classification project: Overview and preliminary results.

[11] Zubi, Zakaria Suliman. "Using some web content mining techniques for Arabic text classification." RECENT ADVANCES on DATA NETWORKS, COMMUNICATIONS, COMPUTERS (2009).

[12] Raheel, Saeed, Joseph Dichy, and Mohamed Hassoun. "The Automatic Categorization of Arabic Documents by Boosting Decision Trees." Signal-Image Technology \& Internet-Based Systems (SITIS), 2009 Fifth International Conference on. IEEE, 2009.

http://dx.doi.org/10.1109/sitis.2009.55

[13] Zahran, Bilal M., and Ghassan Kanaan. "Text Feature Selection using Particle Swarm Optimization Algorithm 1." (2009).

[14] Kanaan, G., Al-Shalabi, R., Ghwanmeh, S., \& Al-Ma'adeed, H. (2009). A comparison of text-classification techniques applied to Arabic text. Journal of the American society for information science and technology, 60(9), 1836-1844.

http://dx.doi.org/10.1002/asi.20832

[15] Gharib, T. F., Habib, M. B., \& Fayed, Z. T. (2009). Arabic Text Classification Using Support Vector Machines. IJ Comput. Appl., 16(4), 192-199.

[16] Harrag, Fouzi, Eyas El-Qawasmah, and Abdul Malik S. Al-Salman. "Comparing dimension reduction techniques for Arabic text classification using BPNN algorithm." Integrated Intelligent Computing (ICIIC), 2010 First International Conference on. IEEE, 2010.

http://dx.doi.org/10.1109/iciic.2010.23

[17] Al-Shargabi, B., Al-Romimah, W., \& Olayah, F. (2011, April). A comparative study for Arabic text classification algorithms based on stop words elimination. In Proceedings of the 2011 International Conference on Intelligent Semantic Web-Services and Applications (p. 11). ACM.

http://dx.doi.org/10.1145/1980822.1980833

[18] Al-diabat, Mofleh. "Arabic text categorization using classification rule mining."Applied Mathematical Sciences 6.81 (2012): 4033-4046.

[19] Hadni, M., Ouatik, S. A., \& Lachkar, A. (2013). Effective Arabic Stemmer Based Hybrid Approach for Arabic Text Categorization. International Journal of Data Mining \& Knowledge Management Process (IJDKP) Vol, 3.

http://dx.doi.org/10.5121/ijdkp.2013.3401

[20] Zaghoul, F. A., and Sami Al-Dhaheri. "Arabic Text Classification Based on Features Reduction Using Artificial Neural Networks." Computer Modelling and Simulation (UKSim), 2013 UKSim 15th International Conference on. IEEE, 2013.

[21] Alghamdi, H. M., \& Selamat, A. (2012, September). Topic detections in Arabic dark websites using improved vector space model. In Data Mining and Optimization (DMO), 2012 4th Conference on (pp. 6-12). IEEE.

http://dx.doi.org/10.1109/dmo.2012.6329790

[22] Ababneh, J., Almomani, O., Hadi, W., El-Omari, N. K. T., \& AlIbrahim, A. (2014). Vector Space Models to Classify Arabic Text. International Journal of Computer Trends and Technology (IJCTT), 7(4), 219-223. 
http://dx.doi.org/10.14445/22312803/IJCTT-V7P109

[23] Al-Eid, R. M. B., Al-Khalif, R. S., \& Al-Salman, A. S. (2010, July). Measuring the credibility of Arabic text content in Twitter. In Digital Information Management (ICDIM), 2010 Fifth International Conference on (pp. 285-291). IEEE.

[24] Alsaleem, S. (2011). Automated Arabic Text Categorization Using SVM and NB. Int. Arab J. e-Technol., 2(2), 124-128.

[25] Al-Shammari, E. T. (2010, November). Improving Arabic document categorization: Introducing local stem. In Intelligent Systems Design and Applications (ISDA), 2010 10th International Conference on (pp. 385-390). IEEE.

[26] Duwairi, R. M. (2007). Arabic Text Categorization. Int. Arab J. Inf. Technol.,4(2), 125-132.

[27] Erkan, G., \& Radev, D. R. (2004). LexRank: Graph-based lexical centrality as salience in text summarization. Journal of Artificial Intelligence Research, 457-479.

[28] Ezzat, H., Ezzat, S., El-Beltagy, S., \& Ghanem, M. (2012, March). Topicanalyzer: A system for unsupervised multi-label arabic topic categorization. In Innovations in Information Technology (IIT), 2012 International Conference on (pp. 220-225). IEEE. http://dx.doi.org/10.1109/innovations.2012.6207736

[29] Harrag, F., El-Qawasmeh, E., \& Pichappan, P. (2009, July). Improving Arabic text categorization using decision trees. In Networked Digital Technologies, 2009. NDT'09. First International Conference on (pp. 110-115). IEEE. http://dx.doi.org/10.1109/ndt.2009.5272214

[30] Jbara, K. (2010). Knowledge discovery in Al-Hadith using text classification algorithm. Journal of American Science, 6(11), 409-419.

[31] Khorsheed, M. S., \& Al-Thubaity, A. O. (2013). Comparative evaluation of text classification techniques using a large diverse Arabic dataset. Language resources and evaluation, 47(2), 513-538. http://dx.doi.org/10.1007/s10579-013-9221-8

[32] Larkey, L. S., Feng, F., Connell, M., \& Lavrenko, V. (2004, July). Language-specific models in multilingual topic tracking. In Proceedings of the 27th annual international ACM SIGIR conference on Research and development in information retrieval (pp. 402-409). ACM. http://dx.doi.org/10.1145/1008992.1009061

[33] Moh'd Mesleh, Abdelwadood. "Feature sub-set selection metrics for Arabic text classification." Pattern Recognition Letters 32.14 (2011): 1922-1929.

http://dx.doi.org/10.1016/j.patrec.2011.07.010

[34] Omar, N., Albared, M., Al-Shabi, A., \& Al-Moslmi, T. (2013). Ensemble of Classification Algorithms for Subjectivity and Sentiment Analysis of Arabic Customers' Reviews. International Journal of Advancements in Computing Technology, 14(5), 77-85.

[35] Thabtah F., Mahazah M., Hadi W. (2008) VSMs with K-Nearest Neighbour to Categorise Arabic Text Data. Proceedings of the International Conference on Machine Learning and Data Analysis 2008 (ICMLDA). Oct. 2008, San Francisco, CA, USA.

[36] Zrigui, M., Ayadi, R., Mars, M., \& Maraoui, M. (2012). Arabic text classification framework based on latent dirichlet allocation. CIT. Journal of Computing and Information Technology, 20(2), 125-140. http://dx.doi.org/10.2498/cit.1001770

[37] Kuang, Qiaoyan, and Xiaoming Xu. "An Improved Feature Weighting Method for Text Classification." AISS: Advances in Information Sciences and Service Sciences 3.7 (2011): 340-346. http://dx.doi.org/10.4156/aiss.vol3.issue7.40

[38] Fattah, Mohamed Abdel. "New term weighting schemes with combination of multiple classifiers for sentiment analysis." Neurocomputing 167 (2015): 434-442. http://dx.doi.org/10.1016/j.neucom.2015.04.051

[39] Salton, Gerard, and Christopher Buckley. "Term-weighting approaches in automatic text retrieval." Information processing \& management 24.5 (1988): 513-523. http://dx.doi.org/10.1016/0306-4573(88)90021-0

[40] Zhang, W., Yoshida, T., \& Tang, X. 2011. A comparative study of TF* IDF, LSI and multi-words for text classification. Expert Systems with Applications,38(3), 2758-2765. http://dx.doi.org/10.1016/j.eswa.2010.08.066

[41] Plötz, T. (2005). Advanced stochastic protein sequence analysis. 